\title{
Multi-event Maintenance Decision-making Model Based on Opportunistic Maintenance Policy
}

\author{
Mengying Zhang ${ }^{1,}$, Gong Wang ${ }^{2, b}$ and Chuan Lv ${ }^{3, c}$ \\ ${ }^{1}$ Technology and Engineering Center for Space Utilization, Chinese Academy of Science, \\ Beijing 100094, China; \\ ${ }^{2}$ Technology and Engineering Center for Space Utilization, Chinese Academy of Science, \\ Beijing 100094, China; \\ ${ }^{3}$ School of Reliability and System Engineering, Beihang University, Beijing 100191, China. \\ azmy@csu.ac.cn, bwanggong@csu.ac.cn Ic@buaa.edu.cn
}

\begin{abstract}
Keywords: Opportunistic maintenance policy, Maintenance decision-making model, Maintenance downtime
\end{abstract}

\begin{abstract}
As one core of modern maintenance theory, the maintenance decision-making theory has become a key research direction with its high precision and systematization. The maintenance decision-making aims to provide a reliable theoretical basis for the exclusion of faults to ensure normal circulation, adjust maintenance plan and treat of benefits, efficiency and economy according to the actual situation. For multi-component complex systems, fault events emerge one after another in a certain time period, a substantial portion of which can be reserved for quite a while and repair then. If the maintenance events are arranged together in a certain opportunity in accordance with opportunistic maintenance policy, maintenance plan can be adjusted and maintenance downtime can be reduced significantly. However, the most part of existing studies focus on pre-established maintenance policies, such as the combination of different preventive maintenance, or combination of preventive maintenance and corrective maintenance. And systematical dynamic maintenance decision-making during real-time operation is rare. Aiming at shortest maintenance downtime, the paper proposes a multi-event opportunistic maintenance decision-making model restricted to maintenance resources and mission requirements, which satisfy dynamic maintenance decision-making demands for complex system. In addition, it will greatly reduce maintenance costs and downtime, while improve system availability.
\end{abstract}

\section{Introduction}

Maintenance problems have always been the significant factor of military, industry and other areas, and directly relate to production quality, reliability of equipment or devices, sustainable development, resource depletion and other issues. According to the actual situation, formulating scientific and reasonable maintenance programs promotes maintenance work continuously towards efficient and economic development [1]. Based on the idea of maintenance, the maintenance decision-making theory use modern scientific decision-making methods to form a relevant theoretical system, and its core issue is the establishment of maintenance model, the optimization of parameters, in-depth analysis and research of the theory and related methods.

Maintenance decision-making has developed since the 1850s and received attention gradually. Current research on maintenance decision-making, in the aerospace, aviation, machinery, especially in the field of marine engineering, has not been given due investment. Some related software is developed, such as US Navy's service resource management system, the United Kingdom's MAINOPT, American RELCODE, PERDEC and AGE / CON software system and so on [2]. The study of this theory has obtained some notable results at home and abroad [3].

For multi-component or complex systems, there are often a number of maintenance events that occur simultaneously. If the original maintenance plan is changed and the maintenance events are 
united together with proper chance, it will greatly shorten maintenance downtime, and reduce maintenance cost.

The most part of existing studies focus on pre-established maintenance policies, such as the combination of different preventive maintenance, or combination of preventive maintenance and corrective maintenance [4]. But systematical dynamic maintenance decision-making during real-time operation is rare [5]. In addition, most research of opportunistic maintenance is currently about fault events which cannot be reserved. Comparatively, opportunistic maintenance policy about reserved fault events gains less attention.

\section{Opportunistic Maintenance Policy}

Opportunistic maintenance policy can be traced back to Age Replacement Policy (ARP) and Block Replacement Policy (BRP) [6]. It doesn't have a standard, normative or acknowledged definition at present. In 1960, the RAND Project defined the opportunistic maintenance as the combination of a preventive maintenance activity and a corrective maintenance activity for reducing maintenance cost first time. Subsequently, the concept is extended into "several maintenance activities are undertaken together", "a preventive maintenance activity and a corrective maintenance are combined" or "the maintenance activities are carried out at an opportunity or in a period of time".

The abstract idea of opportunistic maintenance is about a maintenance opportunity that may arise in a system. There are three cases to apply that "opportunity": first, the system is down because of corrective maintenance activities; second, the system is down as a result of preventive maintenance activities; the last one is the idle time of the operating system or the interval between tasks.

Considering the concept of "opportunity" mentioned above, the best description of opportunistic maintenance is, under the target of avoiding future failures and reducing equipment downtime, the repair or replacement activities for some components conducted in an opportunity when another component need to maintain in the same system [7].

In summary, the paper concludes the definition of opportunistic maintenance is, aiming at reducing failures, shorten system downtime and costs, the maintenance plan and arrangement to unite and optimize some maintenance activities at an appropriate opportunity.

Opportunistic maintenance can analysis and conduct fault events timely, which can ensure the reliability and safety of system and improve the availability by adjusting maintenance plan. What's more, it can reduce maintenance downtime, costs and nonscheduled maintenance frequency. In addition, the policy helps plan support resources scientifically by reducing spare parts, support facility and maintenance personnel. But the opportunistic maintenance will bring longer maintenance time in a combination operation, more expensive management cost and lower authenticity for actual decision-making affect.

\section{Multi-event Opportunistic Maintenance Decision-making Model}

Demand analysis. When many fault events emerge in a system at a time, maintenance decision-making reasoning process is utilized to seek for a good maintenance policy. The paper takes two fault events as example to show the demand analysis process of opportunistic maintenance decision-making, as shown in Fig. 1.

1) Receive fault event signals from dynamic information;

2) Determine the attribute of event by maintenance opportunity adjustment: reserved fault events and immediate fault events (cannot be reserved);

3) Acquire a reserved event from the maintenance event database;

4) Divide the attribute of multi-event in two kinds: two reserved fault events, a reserved fault event and an immediate fault event.

5) Apply opportunistic maintenance policy for the two reserved fault events, if the maintenance decision-making model has solutions, repair the two events together in the proper time; if the model has no solutions, reserve both of the events and send them back to maintenance event database. 
6) For the reserved fault event and the immediate event, if the model has solutions, combine the two events together and repair them immediately; otherwise, repair the immediate event alone and send the other back to the database.

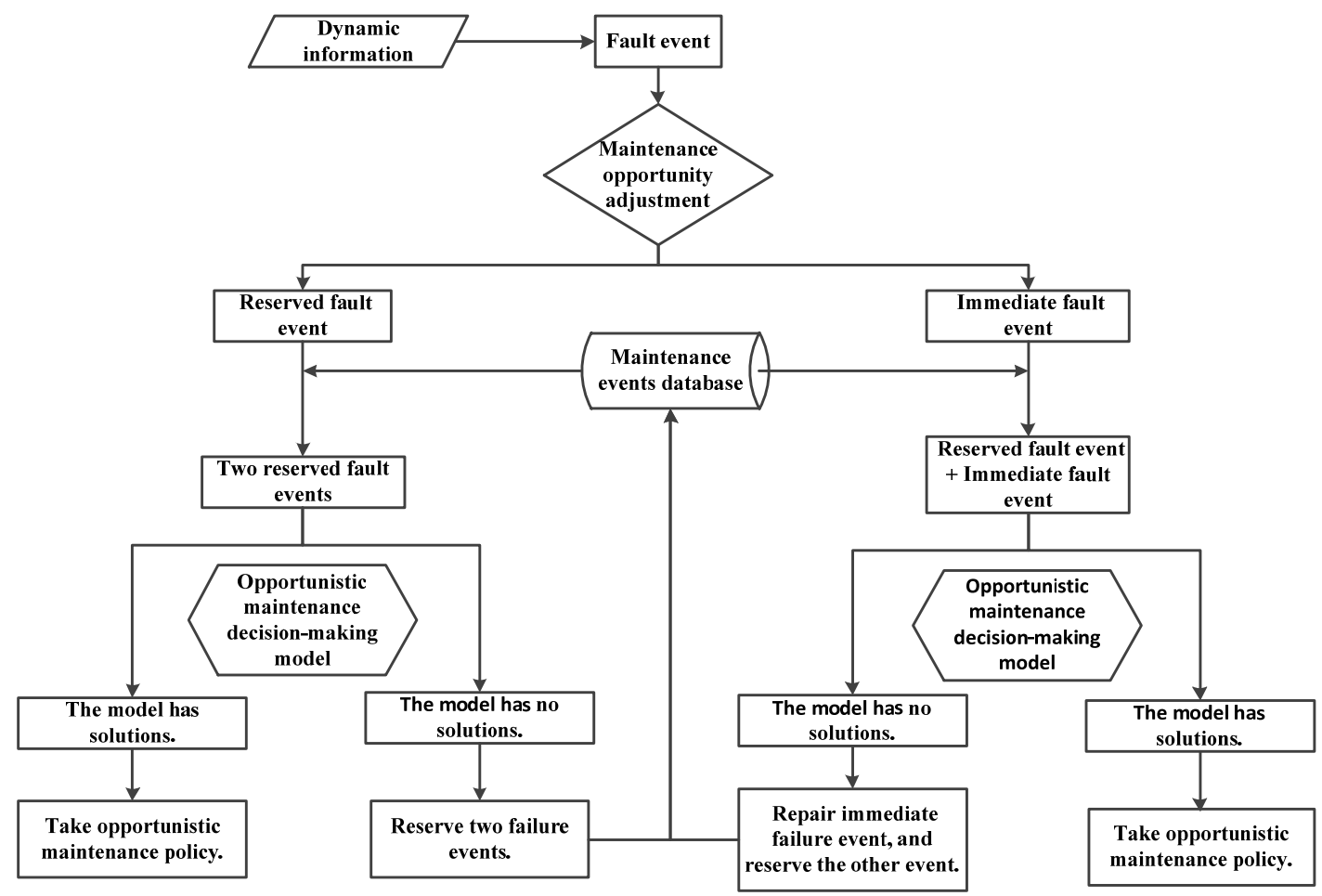

Fig. 1 Demand analysis for opportunistic maintenance decision-making

This paper mainly discusses opportunistic maintenance policy for two reserved fault events, which can find out the optimal opportunity to unite two reserved events.

Maintenance downtime of singular fault event. Maintenance downtime of the fault event is not fixed but changes gradually over time. When the fault occurs just recently, maintenance downtime is generally considered to remain the same; during the reserved fault component becoming more severe and causing deterioration of other parts of the system as time goes by, the downtime increases gradually; when the fault lies over until a certain extent, the deterioration impact on the fault system and associated components has played to the maximum, and its maintenance downtime doesn't change but remain at a level.

On the premise of fault being reserved, the change law of maintenance downtime as time goes by is not discovered deeply. The maintenance downtime is simplified and assumed as the curve shown in Fig. 2:

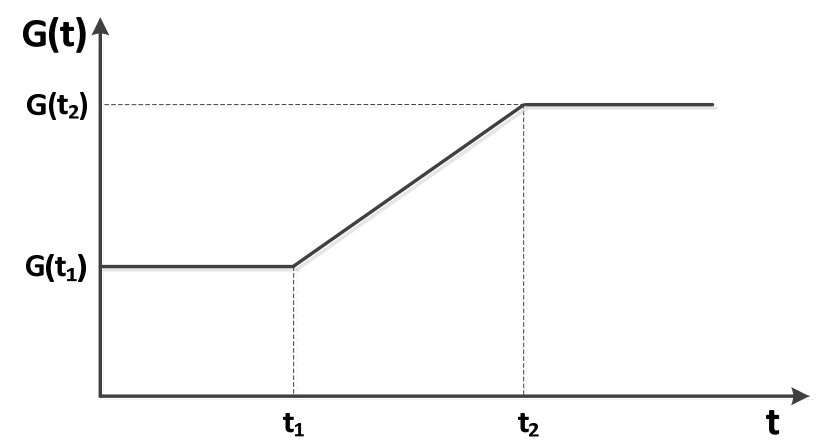

Fig. 2 Maintenance downtime function

The function of downtime is expressed as Formula (1): 


$$
G(t)= \begin{cases}G\left(t_{1}\right), & 0 \leq t<t_{1} \\ \frac{G\left(t_{2}\right)-G\left(t_{1}\right)}{t_{2}-t_{1}} t+\frac{G\left(t_{1}\right) t_{2}-G\left(t_{2}\right) t_{1}}{t_{2}-t_{1}}, & t_{1} \leq t \leq t_{2} \\ G\left(t_{2}\right), & t>t_{2}\end{cases}
$$

Maintenance downtime reduced under opportunistic maintenance policy. With a possible opportunity, unite the maintenance events together, which can directly reduce maintenance downtime. The reduced maintenance downtime is expressed as $T^{\prime}(t)$, and its value can be determined by ergodic method.

Firstly, resolve maintenance downtime $T^{1}(t)$ and $T^{2}(t)$ of the two events into the smallest time units and represent them by two-dimensional arrays as Formula $(2,3)$, where the elements in the first column $T_{x} / T_{y}$ are on behalf of the attributes of time and the elements in the second column $m_{i} / n_{j}$ are the values of time.

$$
\begin{aligned}
T^{1}(t) & =\left[\begin{array}{cc}
T_{1} & m_{1} \\
\cdots & \cdots \\
T_{x} & m_{x} \\
\cdots & \cdots \\
T_{i} & m_{i}
\end{array}\right] \\
T^{2}(t) & =\left[\begin{array}{cc}
T_{1} & n_{1} \\
\cdots & \cdots \\
T_{y} & m_{y} \\
\cdots & \cdots \\
T_{j} & n_{j}
\end{array}\right]
\end{aligned}
$$

Secondly, use ergodic method to find out the same rows between $T^{1}(t)$ and $T^{2}(t)$, which means their same time units reduced during opportunistic maintenance. If $\left[\begin{array}{ll}T_{c} & m_{c}\end{array}\right]=\left[\begin{array}{ll}T_{c} & n_{c}\end{array}\right]$, it indicates that the two smallest time unit coincide, and mark $m_{c}$ as $m_{c}^{*}$.Then, $T^{\prime}(t)$ can be expressed as Formula(4):

$$
T^{\prime}(t)=\sum_{1}^{i} m_{x}^{*}
$$

The substance of two-dimensional array is 'array of arrays'. When two two-dimensional arrays have the same data format, ergodic method can be utilized to find out the same row/column. Take each row/column of the first array successively, compare it with each row/column of the other array, and the same row/column can be found out. For example, there are two-dimensional arrays M and N, as shown in Formula $(5,6)$ :

$$
M=\left[\begin{array}{ll}
a & 10 \\
b & 15 \\
c & 20 \\
\text { d } & 15 \\
\text { e } & 25
\end{array}\right]
$$




$$
\mathrm{N}=\left[\begin{array}{ll}
a & 20 \\
b & 15 \\
c & 30 \\
d & 15 \\
\text { e } & 30
\end{array}\right]
$$

Find out the same row between them by ergodic method, and use $\mathrm{C} \#$ to realize it:

static void Main(string[ ] args)

\{

string[,] $\mathrm{M}=$ new string[1000,2];

string[,] $\mathrm{N}=$ new string[1000,2];

int $\mathrm{i}=0, \mathrm{j}=0$;

for $(\mathrm{i}=0 ; \mathrm{i}<$ M.GetLength $(0) ; \mathrm{i}++)$

\{

for $(j=0 ; j<N . G e t L e n g t h(0) ; j++)$

\{

if $(\mathrm{M}[\mathrm{i}, 0]==\mathrm{N}[\mathrm{j}, 0])$

\{

if $(\mathrm{M}[\mathrm{i}, 1]==\mathrm{N}[\mathrm{j}, 1])$

\{

Console.Write(M[i,0] + "," + M[i,1]);

Console.WriteLine();

\}

else

\{

continue;

\}

\}

else

\{

continue;

\}

\}

\}

As calculation result shown, [b 15] and $[\mathrm{d} 15]$ are the same rows, so $T^{\prime}(t)$ is 30 .

Incorporate maintenance downtime of two fault events. When opportunistic maintenance policy is applied for two fault events, and the two maintenance activities are united into one, the incorporate maintenance downtime is expressed as Formula (7):

$$
T^{0}=T^{1}(t)+T^{2}(t)-T^{\prime}(t)
$$

Where, $T^{0}$ is the incorporate maintenance downtime of two fault events;

$T^{1}(t)$ is the maintenance downtime of the first event;

$T^{2}(t)$ is the maintenance downtime of the other event;

$T^{\prime}(t)$ is the reduced maintenance downtime under opportunistic maintenance policy, namely the same time units of two maintenance events.

Opportunistic maintenance decision-making model for two reserved fault events. To construct the maintenance decision-making model for two reserved fault events, there are some constraints to be considered as shown in Table 1: 
Table 1 Modeling constraints

\begin{tabular}{|c|c|c|}
\hline No. & Symbol & Description \\
\hline 1 & $\begin{array}{l}P(t), Q(t) \\
E(t), F(t)\end{array}$ & $\begin{array}{l}\text { Separately represent the number of personnel and technical level, spare parts } \\
\text { support, support facility and support device required at the time } \mathrm{T} \text { (the other } \\
\text { support resources are not directly related to opportunistic maintenance) }\end{array}$ \\
\hline 2 & $\begin{array}{l}P^{0}(t), Q^{0}(t) \\
E^{0}(t), F^{0}(t)\end{array}$ & $\begin{array}{l}\text { Separately represent the number of personnel and technical level, spare parts } \\
\text { support, support facility and support device available at the time } \mathrm{T}\end{array}$ \\
\hline 3 & $T_{\text {task }}$ & $\begin{array}{l}\text { The component need to perform task at } T_{\text {task }} \text {, thus the fault event can be reserved } \\
\text { until this latest time. }\end{array}$ \\
\hline 4 & $T_{\max }$ & $\begin{array}{l}\text { If repair is not carried out before } T_{\max } \text {, it will lead to serious consequences, such } \\
\text { as the system breakdown and casualties. }\end{array}$ \\
\hline 5 & $T^{0}{ }_{\text {limit }}$ & Represent the threshold of maintenance downtime by managers. \\
\hline
\end{tabular}

To conclude, aiming at shortest maintenance downtime, the maintenance decision-making model for two reserved fault events with the maintenance opportunity as variable is as Formula (8):

$$
\begin{cases}\min & T^{0}(t)=T^{1}(t)+T^{2}(t)-T^{\prime}(t) \\ \text { s.t. } & S\{P(t), Q(t), \quad F(t), \quad E(t)\} \subseteq S\left\{P^{0}(t), Q^{0}(t), F^{0}(t), E^{0}(t)\right\} \\ \text { s.t. } & 0 \leq t \leq \min \left\{T_{\text {task }}, T_{\max }\right\} \\ \text { s.t. } & T^{0}(t) \leq T_{\text {limit }}^{0}\end{cases}
$$

The solution process for the model is shown in Fig. 3:

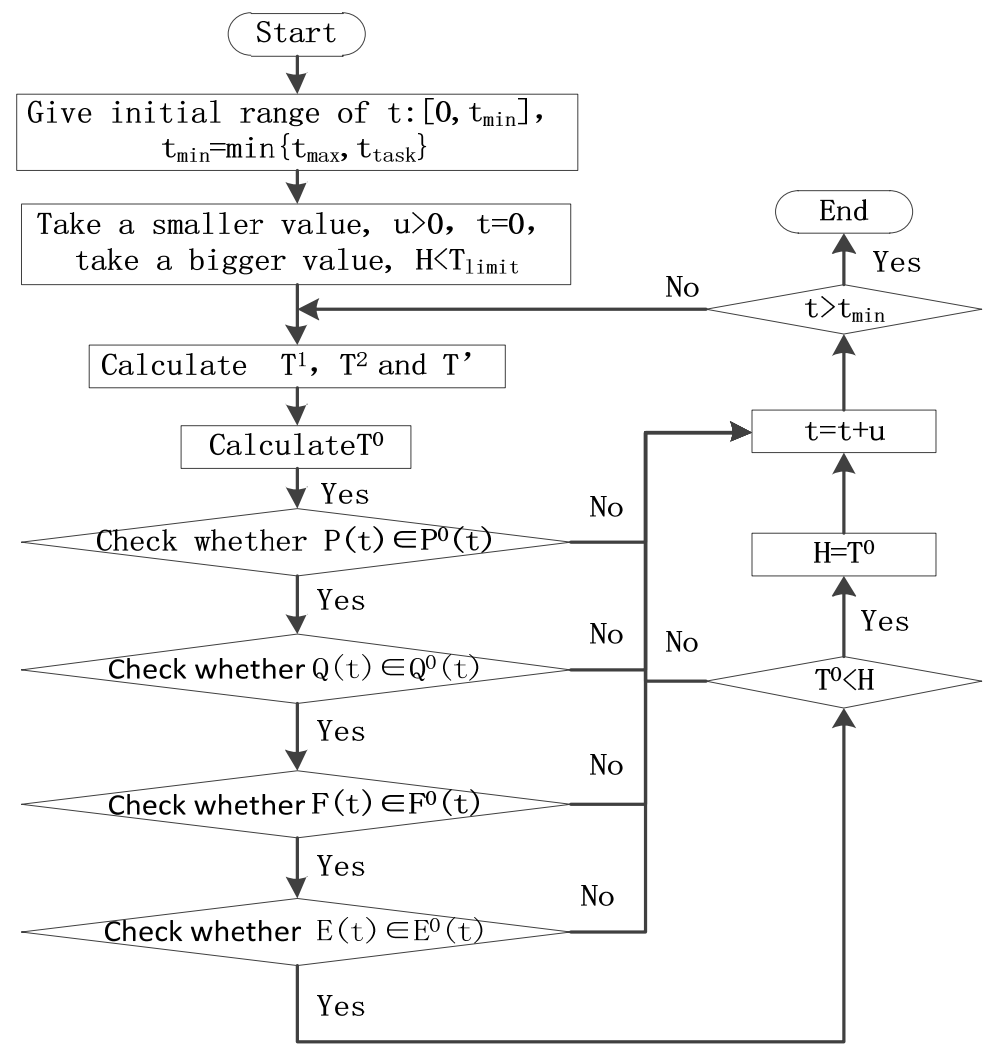

Fig. 3 Solution process for the model 


\section{Example}

Take a SD504 cut slicing machine in the slicing workshop of a cigarette factory as example. Aiming at the shortest maintenance downtime, opportunity maintenance decision-making model is utilized to calculate the best maintenance opportunity and work out an optimal maintenance plan for two reserved fault events of the machine.

Fault event analysis. There are two fault events occurred on a slicing machine in the morning, Table 2 is the fault events list.

Table 2 Fault events list

\begin{tabular}{ccccccc}
\hline No. & Fault time & Fault appearance & Fault reason & $\begin{array}{c}\text { Longest } \\
\text { reserved } \\
\text { time }\end{array}$ & Measure & $\begin{array}{c}\text { technical level } \\
\text { of personnel }\end{array}$ \\
\hline A & $\begin{array}{c}2012-04-12 \\
12: 30: 40\end{array}$ & $\begin{array}{c}\text { Tobacco slicing } \\
\text { non-uniform }\end{array}$ & $\begin{array}{c}\text { Slicing } \\
\text { blade } \\
\text { abrasion } \\
\text { Feed }\end{array}$ & 2 hours & replacement & $\begin{array}{c}\text { No lower than } \\
\text { level } 4\end{array}$ \\
B & $\begin{array}{c}\text { 2012-04-12 } \\
11: 00: 10\end{array}$ & $\begin{array}{c}\text { Slicing blade feed } \\
\text { abnormal }\end{array}$ & $\begin{array}{c}\text { segment } \\
\text { abrasion }\end{array}$ & 4 hours & replacement & $\begin{array}{c}\text { No lower than } \\
\text { level 3 }\end{array}$ \\
\hline
\end{tabular}

Working hours is $8: 00$ to $12: 00$ and $13: 00$ to $17: 00$ in the factory. 12:00 to13:00 is the break time and there is only a 4 level personnel on duty in maintenance department. Because an important task will be performed at 14:00, the machine must be repaired before 14:00. In addition, the singular maintenance downtime is fixed no more than 60 minutes.

Maintenance operation analysis. Maintenance activities for slicing machine should be conducted by $<$ SD504 Slicing machine maintenance and service handbook $>$.

Table 3 is the maintenance time analysis of fault event A. $T_{1}^{d}$ to $T_{6}^{d}$ is the time for shuting down, turning off electricity and other preparation work; $T_{7}^{d}$ to $T_{12}^{d}$ it the time for removing slicing blade; $T_{13}^{d}$ to $T_{23}^{d}$ is the time for installing new blade; $T_{24}^{d}$ and $T_{25}^{d}$ is the time for regain work. $T_{\Delta}^{d}$ is called maintenance downtime growth factor. As the maintenance downtime changes over time, it will increase from 30 minutes at 12:30 to 35 minutes at 13:10 for event $\mathrm{A}$, and the increased 5 minutes is the maintenance downtime growth factor. $T^{d}$ is the total maintenance time. The maintenance activity is to replace the slicing blade, shown as Fig. 4.

Table 4 is the maintenance time analysis of fault event B. $T_{1}^{d}$ to $T_{25}^{d}$ for event B is the same with event A. $T_{1}^{s}$ to $T_{6}^{s}$ is the time for replacing feed segment. $T_{\Delta}^{s}$ is the maintenance downtime growth factor and $T^{s}$ is the total maintenance time for event $\mathrm{B}$. The maintenance activity is to replace the feed segment, as Fig. 5.

Table 3 Maintenance time analysis of fault event A

\begin{tabular}{ccccccccccccccc}
\hline Time code & $T_{1}^{d}$ & $T_{2}^{d}$ & $T_{3}^{d}$ & $T_{4}^{d}$ & $T_{5}^{d}$ & $T_{6}^{d}$ & $T_{7}^{d}$ & $T_{8}^{d}$ & $T_{9}^{d}$ & $T_{10}^{d}$ & $T_{11}^{d}$ & $T_{12}^{d}$ & $T_{13}^{d}$ & $T_{14}^{d}$ \\
Value(min) & 0.5 & 0.5 & 0.5 & 0.5 & 2 & 1 & 1 & 1 & 2 & 1 & 2.5 & 2 & 1.5 & 1 \\
\hline Time code & $T_{15}^{d}$ & $T_{16}^{d}$ & $T_{17}^{d}$ & $T_{18}^{d}$ & $T_{19}^{d}$ & $T_{20}^{d}$ & $T_{21}^{d}$ & $T_{22}^{d}$ & $T_{23}^{d}$ & $T_{24}^{d}$ & $T_{25}^{d}$ & $T_{\Delta}^{d}$ & $T^{d}$ \\
Value(min) & 1 & 1 & 1 & 1.5 & 2 & 2 & 0.5 & 2 & 0.5 & 0.5 & 1 & 5 & $30-35$ \\
\hline
\end{tabular}


Table 4 Maintenance time analysis of fault event B

\begin{tabular}{ccccc}
\hline Time code & $T_{1}^{d}$ & $T_{2}^{d}$ & $T_{3}^{d}$ & $T_{4}^{d}$ \\
Value(min) & 0.5 & 0.5 & 0.5 & 0.5 \\
\hline Time code & $T_{12}^{d}$ & $T_{1}^{s}$ & $T_{2}^{s}$ & $T_{3}^{S}$ \\
Value(min) & 2 & 0.5 & 1 & 1 \\
\hline Time code & $T_{17}^{d}$ & $T_{18}^{d}$ & $T_{19}^{d}$ & $T_{20}^{d}$ \\
Value(min) & 1 & 1.5 & 2 & 2 \\
\hline Clamping device & & & & \\
\hline
\end{tabular}

Fig. 4 Replace slicing blade

$\begin{array}{llllll}T_{6}^{d} & T_{7}^{d} & T_{8}^{d} & T_{9}^{d} & T_{10}^{d} & T_{11}^{d}\end{array}$

$\begin{array}{ccccccc}2 & 1 & 1 & 1 & 2 & 1 & 2.5 \\ T_{4}^{S} & T_{5}^{S} & T_{6}^{s} & T_{13}^{d} & T_{14}^{d} & T_{15}^{d} & T_{16}^{d}\end{array}$

$\begin{array}{llll}2 & 1 & 4.5 & 1.5\end{array}$

$\begin{array}{lllllll}T_{21}^{d} & T_{22}^{d} & T_{23}^{d} & T_{24}^{d} & T_{25}^{d} & T_{\Delta}^{s} & T^{s}\end{array}$

0.5

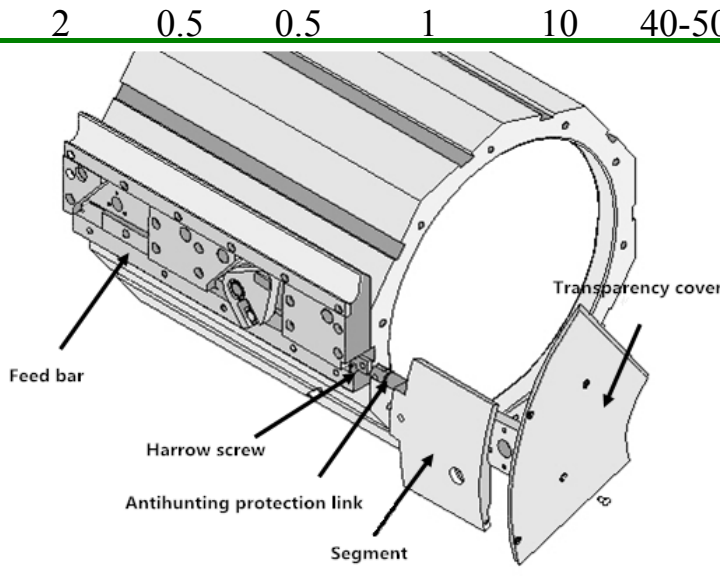

Fig. 5 Replace feed segment

Opportunistic maintenance decision-making. The case takes $12: 30$ as the starting time to carry out maintenance decision-making. 10):

The maintenance downtime function for the event A and B are separately expressed as Formula (9,

$$
\begin{gathered}
G^{1}(t)= \begin{cases}30, & 0<t<20 \\
\frac{1}{4} t+25, & 20 \leq t \leq 40 \\
35, & x>40\end{cases} \\
G^{2}(t)= \begin{cases}40, & 0<t<5 \\
\frac{1}{2} t+\frac{75}{2}, & 5 \leq t \leq 25 \\
50, & x>25\end{cases}
\end{gathered}
$$

The reduced maintenance downtime is calculated by ergodic method, and the function is as Formula (11):

$$
T^{\prime}(t)= \begin{cases}0, & 0 \leq t \leq 30 \\ 30, & t>30\end{cases}
$$

Thus, the incorporate maintenance downtime function is as Formula (12):

$$
T^{0}(t)=G^{1}(t)+G^{2}(t)-T^{\prime}(t)= \begin{cases}70, & 0 \leq t<5 \\ \frac{1}{2} t+\frac{135}{2}, & 5 \leq t<20 \\ \frac{3}{4} t+\frac{125}{2}, & 20 \leq t<25 \\ \frac{1}{4} t+75, & 25 \leq t<30 \\ \frac{1}{4} t+45, & 30 \leq t<40 \\ 55, & t \geq 40\end{cases}
$$


Above all, the multi-fault-event opportunistic maintenance decision-making model is shown as Formula (13):

$$
\begin{cases}\text { min } & T^{0}(t) \\ \text { s.t. } & S\{P(t), Q(t), \quad F(t), \quad E(t)\} \subseteq S\left\{P^{0}(t), Q^{0}(t), F^{0}(t), E^{0}(t)\right\} \\ \text { s.t. } & 0 \leq t \leq \min \{120,150\} \\ \text { s.t. } & 0 \leq t \leq 90 \\ \text { s.t. } & T^{0}(t) \leq 60\end{cases}
$$

The model solution is $t=30 \mathrm{~min}$, and $T_{\text {min }}^{0}(t)=52.5 \mathrm{~min}$. Namely, if opportunistic maintenance activities are conducted at 30 minutes, the incorporate maintenance downtime for the events $\mathrm{A}$ and $\mathrm{B}$ is the minimum value 52.5 minutes.

\section{Summary}

In the case of a complex system with many fault events, the paper utilizes opportunistic maintenance policy to make decisions for two reserved events. Targeted at shortest maintenance downtime, an opportunistic maintenance decision-making model is constructed in consideration of maintenance resources and task requirements. And the model is demonstrated by a slicing machine in the end. The opportunistic maintenance decision-making model satisfies dynamic decision-making demand in real-time operation and directly reduces maintenance downtime. However, actually there are more complex circumstances and constraints, and the model for other types of maintenance events should also be studied further.

\section{References}

[1] Zuo HF, Cai J, et al. Maintenance decision-making theory and methods[M]. Aviation industry Press, 2008.

[2] Faccio M, Persona A, Sgarbossa F, et al. Industrial maintenance policy development: A quantitative framework[J]. International Journal of Production Economics, 2014, 147: 85-93.

[3] Li CY, Xu MQ, Guo S, et al. Research and prospect of maintenance decision-making[J]. Thermal Turbine, 2008, 37(1): 46-50.

[4] Zhou X, Xi L, Lee J. Opportunistic preventive maintenance scheduling for a multi-unit series system based on dynamic programming[J]. International Journal of Production Economics, 2009, 118(2): 361-366.

[5] Pham H, Wang H. Imperfect maintenance[J]. European Journal of Operational Research, 1996, 94(3): 425-438.

[6] Huang CG. Equipment Maintenance Policy Model analysis and decision support[D]. Huazhong University of Science and Technology, 2006, 17-19.

[7] Chang Q, Ni J, Bandyopadhyay P, et al. Maintenance opportunity planning system[J]. Journal of manufacturing science and engineering, 2007, 129(3): 661-668. 